\title{
Foreword - Exotic Stars as Links to ...
}

\author{
R. E. Wilson \\ Astronomy Department, University of Florida
}

The world is full of surprises - one cannot know what will become useful, even critical, in understanding a given area. That exotic stars are intrinsically fascinating, yet strongly connected to other astrophysical areas, was accordingly a central theme of the Colloquium. Examples come easily - we would like to understand the cosmic gamma-ray bursts, and there we need stellar evolution theory and evolution theory needs exotic stars. SS 433 is an exotic star with properties of a miniature Active Galactic Nucleus (AGN). Supernova 1987A is an exotic star that probes the late stages of stellar evolution. Stars are supposedly well understood, so today's trends are in pursuits such as distant galaxies, really distant galaxies, and - going all the way - cosmology. Much work in those areas is built upon observational statistics of stars in our Galaxy, but statistical knowledge of common stars is not enough, as there is need to understand the processes and mechanisms of stellar evolution. That is, there is more to do than gather nearby statistics if we want to model stellar populations, variable stars and star formation histories in distant galaxies. The well known reason is that stellar evolution depends on chemical abundances and therefore on cosmic time so, with consequent distance dependence, nearby statistics apply only to our present cosmological age. Direct examples of these links include work on the theory of Type Ia supernovae, Cepheid light curves, and HR diagram distributions, all with dependence on composition. However we also have the continual flow of indirect contributions (i.e. not specifically addressed to cosmological consequences) - from normal stars of course - but also from the non-normal, including exotic and ultra-exotic stars. Perhaps most basic is that evolved cores become exposed to observation in a wide variety of exotic stars and thereby give explicit information on otherwise invisible conditions.

Exotic star research typically examines a star or star system intensively, often with quite adequate signal, while distant Universe research extracts rough information from faint smudges of light. Clearly we have two quite distinct research worlds, with the potential bridge being the field of stellar evolution. Work on the distant Universe needs solid underpinning from stellar evolution theory if it is to deal with cosmic-era effects, but evolution theory needs checks from exotic stars to stay on course. One could easily regard the distant Universe and exotic stars as unconnected, given that the latter are too rare to influence large scale statistics, but exotic stars are indispensible guides for evolution theory, and we must get evolution theory right - including cosmic era effects - if we are to get the early Universe right.

Exotic star problems might be divided into three levels. First we have level 1 of, "What is going on?" with sub-division into 1a, "Does anyone have an idea?" and 1b, "It's probably ... but ...." Examples could be $\eta$ Carinae and many symbiotic stars. In level 2 most essential phenomena are identified but 
specifics are blurry. Examples could be $\beta$ Lyrae, $\epsilon$ Aurigae, and FG Sagittae. Level 3 is for sub-exotics, where we have a quantified model that predicts most observations reasonably well and can go on to work on the lesser issues. These stars were true exotics at earlier times. Examples would be Miras and the many RS CVn and Algol binaries.

At all levels of exotics, a major role is played by chemical composition. One Colloquium contribution after another had composition as a major issue or the major issue, as in CW Leo, Przybylski's star, V652 Her, and FG Sge, so interplay of composition and evolution alone gives sufficient justification for exotic star astronomers coming together. Then we have the population synthesis connection, the gamma burst connection, and the AGN connection. In reality, the Exotic Stars meeting grew out of accidental dinner seating next to Andi Burkert after a Cambridge Institute of Astronomy colloquium. Stimulated by under two bottles of wine, the issue arose - what sort of meeting would fill a genuine need, as opposed to following a popular trend, and had not been done before? Well, most things on stars would be safely non-trendy, but what about the not done before part? Indeed meetings on kinds of observations of stars, physics of stars, evolution of stars, classes of strange and normal stars, and particular strange stars have been held, even repeatedly. But had there been a meeting on diverse individual strange stars? We could not remember one. Initial reaction from various IOA members and visitors over following weeks was remarkably positive and, although the winding road to the actual gathering would be a long story, the topic struck a chord with the community of star workers and led to sponsorship by the Heidelberg Max Planck Institute for Astronomy and Florida International University in addition to the IAU. Certainly a remarkable variety of topics was covered. Attendance was curtailed somewhat by travel problems, partly resulting from events of late 2001, but enthusiasm among attendees attested to the need for a forum on Exotic Stars, as will be evident in the pages to follow. 